\title{
STATISTICAL UTILIZATION OF MATERNITY AND CHILD WELFARE RECORDS
}

\author{
BY \\ ENID CHARLES \\ From the Central Statistical Office, City of Birmingham, and the Department of \\ Social Medicine, University of Birmingham
}

\section{INTRODUCTION}

The collection and analysis of statistical material relating to mortality and morbidity has long been an important part of the functions of Public Health Authorities. Much of the data analysed at a national level is transmitted through the local authority and reciprocal arrangements have developed whereby statistical analysis of its own problems by each authority runs pari passu with centralized statistics for the country as a whole. In the field of child welfare, the rapid fall in infant mortality has shifted emphasis on the one hand to the importance of the antenatal period in relation to the hard core of neonatal mortality, and on the other to less immediately lethal aspects of child health. In both these fields local authorities have responsibilities which call for periodic assessment.

The characteristics of Maternity and Child Welfare data of most interest to vital statisticians are that they cover all or nearly all of a complete population in the demographic sense, and that they record first-hand observations of events and conditions which might otherwise be missed. The data are, however, frequently less precise than can be obtained by trained investigators. A brief account of the methods now being adopted by the Maternity and Child Welfare Department of the City of Birmingham will illustrate the scope and limitations of Public Health material, and may be of interest to medical statisticians engaged in comparable tasks.

The Maternity and Child Welfare Department, through its frequent contact with most of the city's mothers and children, has studied intensively aspects of neonatal mortality and the care of immature infants; but a considerable corpus of information collected by midwives and health visitors regarding surviving children has not hitherto been available in a form amenable to systematic analysis. To utilize more effectively all existing data and to meet other administrative needs, the Senior Assistant Medical Officer of Health for Maternity and Child Welfare sought the assistance of the Central Statistical Office in re-designing some of the forms, and in planning a mechanized record system. Thus on January 1, 1949, was inaugurated a new system of records intended to make more readily available the medical and social histories of all children in the city under the age of 5 years. No radical changes in departmental policy or organization were involved, since health visitors were already visiting about 98 per cent. of all births to Birmingham residents at 
least once, and no new items of information were asked for; but a considerable effort was made to secure greater precision of definition and more complete coverage.

The Central Statistical Office was materially assisted by investigations carried out in Birmingham and Smethwick by the Department of Social Medicine of the University of Birmingham, as also by the new medical documents designed by the Department of Medical Statistics.

\section{OBJeCtives}

Two chief motives prompted a change in the form of the records kept. In the first place, it had long been felt both by School Medical Officers and by the Maternity and Child Welfare Department that a link between the department's knowledge of the child's pre-school medical history and its school medical record is highly desirable. This presupposes the availability of one document recording the principal items of the pre-school history in a form suitable for transmission to the school when the child passes out of the care of the department. Secondly, observations on children surviving beyond the first birthday have not been hitherto amenable to statistical analysis, being in fact no longer available after the care of the individual child has ceased.

When the principal items in the antenatal record and subsequent history of the child up to 5 years old are codified in such a way that they can be transferred to a punch card, material for several new lines of enquiry becomes available.

(i) When the antenatal care and type of care at delivery can be directly related to the subsequent history of the infant, a more precise assessment of the effects of public provision on infant welfare can be made.

(ii) The same type of assessment can be made of social conditions affecting the child.

(iii) The direct association between events in the antenatal period and during labour with the subsequent history of the baby permits a relatively novel approach to certain aspects of infant mortality and morbidity.

Needless to say, mortality rates alone do not give a complete picture of the significance of a pathological condition. In addition, fatality, absentee, and disablement rates are required so that resulting deaths are assignable to total incidence of the condition. Evidently, the field in which such an approach is possible is limited to conditions whose total ascertainment is practicable in a given population, e.g. communicable diseases, and army medical statistics. Maternal conditions, e.g. toxaemia, difficult labour, etc., are frequently recorded as the main or a contributory cause of stillbirths and neonatal deaths, but there has been little opportunity to examine the fatality associated with such events. In so far as the department's records can be extended to cover the whole city population, it will be possible to compute fatality rates for most of the occurrences believed to be associated with increased infant mortality. Preliminary rates of this type have been given here or elsewhere for certain maternal diseases and complications of pregnancy, Rhesus serum incompatibility, and duration of labour.

(iv) Local variations within the city with respect to mortality and morbidity in relation to the use made of public health facilities can be studied intensively, thus assisting in the formulation of programmes for future development.

(v) The punch-card file can provide the basic data required for any ad.hoc investigation, whether initiated at a national or at a local level, thus reducing considerably the work 
involved. It is also easy to draw a random sample of any type required for an investigation of limited scope.

(vi) When detailed breakdowns are needed or comparatively rare conditions are being studied, numbers for a single year are too small even in a city with over a million inhabitants. Routine collection of data year by year will make it possible eventually to investigate any question on the basis of adequate numbers since the data are always available for further study.

(vii) Since all aspects of mortality and morbidity can be classified by age and parity of mother, it is possible to examine trends from year to year free from the effects of changing age and parity. This is particularly important at the present time when the proportion of first births is high and the numbers of births may rise or fall rapidly with a consequent unstable maternal age and sex composition.

\section{Documents AND Sources}

What follows, though tentative, may be of interest to workers in Public Health Departments as illustrative of types of information which can be salvaged with comparatively little effort by appropriate design of the Health Visitor's Record Card, without imposing on the health visitor the burden of recording items other than those the record as formerly designed should include. The appendix exhibits both the old card and the new one which has been introduced in order to exploit mechanical aids for statistical analysis (Figs 3 to 6).

As far as possible, the items are in self-coded form. Where this is not practicable, space is left for office coding of:

(a) occupation

(b) cause of death on death certificate, for which office coding already existed

(c) a few simple arithmetical codes.

The last-named type of departure from the self-coding principle is intended to save the health visitor's very valuable time, the extra office work involved being small.

The first statistical page of the card contains basic information concerning the baby, the mother, and the family. Certain items are completed at the office before the card is sent out, from the notification of birth, and the rest is completed by the health visitor at her first or second visit.

The second page contains:

(a) the antenatal record, obtained from the clinic or hospital responsible for antenatal care,

(b) particulars of the delivery and of the baby's condition during the first two weeks after birth.

All medical items are intended to be recorded by the doctor, nurse, or midwife directly responsible, or to be copied from the doctors' record at the antenatal clinic. In no case is a layman's statement accepted about a pathological condition. To secure completion of this second page, a copy is circulated to those concerned, and the particulars afterwards transferred to the visitor's Record Card. Though copying is to be deprecated, it is here unavoidable, since the Record Card is required by the health visitor soon after the child's birth, and to circulate it might cause delay. 
The third and fourth statistical pages summarize in codifiable form the main features of the child's history up to 5 years. The difficulties of reducing an historical record to statistical form are well known. In the present case, the occurrences of a few developmental events are recorded in six-monthly periods, and the remainder of the space summarizes the child's physical state and living conditions at 1 year and at 5 years. The health visitor is the main source for all information subsequent to birth. Links with hospitals and residential institutions are under review.

The Record Card is returned to the office for coding and punching on three occasions:

(a) as soon as the basic information on the first page has been secured, usually from

3 weeks to 2 months after birth;

(b) at 1 year;

(c) at 5 years.

In the event of death after 4 weeks, or of removal, the relevant facts are inserted and the card returned; this renders unnecessary two forms previously in use, that for deaths between 4 weeks and 1 year, and that for the follow-up of premature births.

The card for neonatal deaths is a replica of the relevant parts of the health visitor's Record Card, together with additional particulars relating to the death. For obvious reasons, most neonatal deaths are not seen by a health visitor, and the responsibility for making returns rests, as it always has done, with the hospital, or with the doctor in attendance. Supplementary information may be sought where possible from the antenatal record or from the health visitor. A stillbirth card on similar lines is in preparation. A summary of the fields of information transferred to an 80-column punch card will be found in the Appendix.

One of the main statistical problems met with in this type of work is administrative. To obtain information at first-hand, data from a variety of sources has to be assembled in one place, and this requires the co-operation of a number of busy people. All those concerned are already overburdened with important duties, and every effort must be made to economize their time and effort. The documents themselves have little claim to originality, and follow in the main existing precedents. Those reproduced in Appendix A are 1950 versions slightly modified from those of 1949 . As the system develops, it should become possible to clarify definitions and to draft cards more precisely suited to public health requirements.

\section{Completeness AND ACCURACY OF ReCORdS}

A punch card is made out for:

(a) every birth occurring in the city,

(b) births to Birmingham residents occurring outside the city,

(c) immigrant children under 5 years old as the department learns of them.

Little except the place of birth is known of births to non-residents, and the main tabulating scheme is concerned only with births to residents. In 1949 there were 20,054 births to resident mothers. Information is complete or very nearly so in. 
respect of residence, plurality, sex, ward, and centre, and whether the birth was domiciliary or institutional. Age of mother was known for $99 \cdot 3$ per cent. of these births, and parity for $99 \cdot 1$ per cent. The small proportion of unknowns seemed to justify their allocation in proportion to the known age and parity cells.

The greater part of the detailed tabulation and analysis relates to a slightly smaller group of 19,711 births in Birmingham and Marston Green Maternity Home to Birmingham residents. Marston Green is included because, though just outside the city limits, it is part of the public provision of maternity care for Birmingham mothers.

Information obtained through health visitors is never likely to be 100 per cent. complete. Response by the mother to a request for information is entirely voluntary and no pressure is ever exerted to obtain answers. This being so, returns for socio-economic agencies (employment of mother during pregnancy, housing, and paternal occupation) of 97 per cent. to 98 per cent. are about the maximum obtainable. The unknown groups are undoubtedly heavily biased in favour of the more prosperous families, but, with such small percentages of unknowns, the error involved is not likely to be serious. Returns from hospitals also depend on voluntary co-operation; this has been forthcoming to a gratifying extent, but may be expected to increase as the systematization of hospital records renders the process of collation less laborious.

The coverage in respect of two most important items, congenital malformations, and birth injuries and diseases in the first two weeks of life, was disappointing. Incompleteness was largely due to observers leaving a blank when there was nothing to report, and such records had perforce to be coded as unknown, but this misunderstanding is gradually being overcome. As in most medical documentation, some taxonomic difficulties were encountered. These problems should themselves prove instructive and the preliminary returns suggest a valuable and comparatively novel field; much is known about congenital malformations, and about early disease and injury leading to death or serious disability, but the incidence of minor defects and pathological conditions is far less familiar.

Complete and accurate returns in respect of the period of gestation are impossible, particularly in the absence of an early pregnancy diagnosis test. In order that the returns may have some meaning, duration is only coded when a definite date for the first day of the last menstrual period has been recorded before the birth of the baby.

Tabulation and analysis has so far dealt only with events up to and immediately after birth. Systematization of the child's later history, though no new information has been sought, is likely to prove more difficult. It may be some time before the material becomes suitable for statistical analysis, but it still has value in providing continuous and compact histories of individual children.

The scope of some of the first year's results is summarized in Table I (overleaf). Items for which the number of unknowns is less than $0 \cdot 1$ per cent. are given as 100 per cent. complete. 
TABLE I

Degree of CoMpleteness of DATA

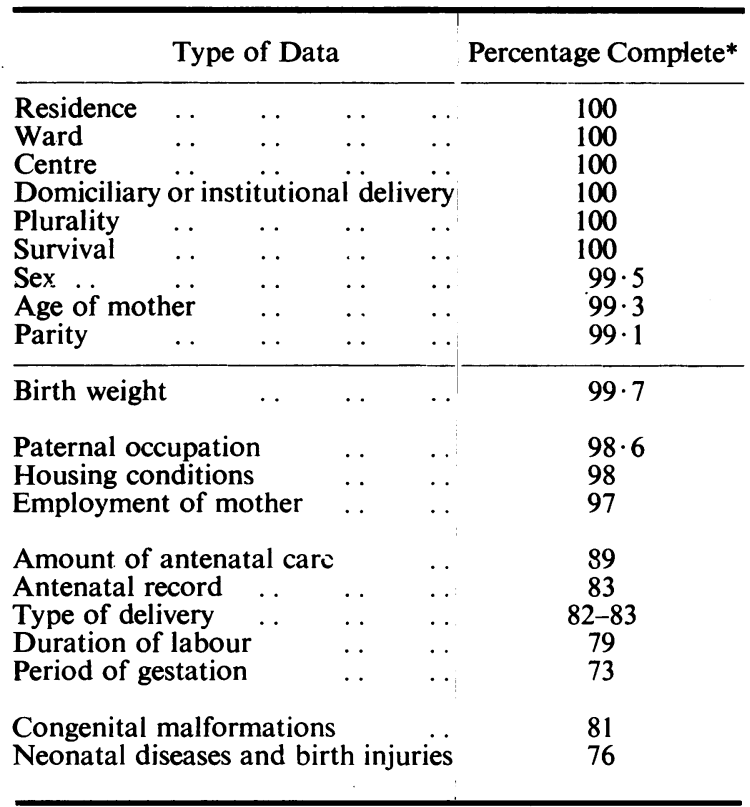

* The first nine items are related to a population of 20,054 live births, the remainder to a population of 19,711 live births.
5. SOME RESUltS OF THE FIRST YEAR's WORK

Since the first year's working of a new record system has been partly experimental, sorting and tabulating was designed to explore a variety of fields to test the validity and usefulness of the data and to ascertain what types of investigation merit more intensive examination. To illustrate the way in which the objectives outlined in Section 2 have been met a selection from the tabular material is presented below with brief comments, and some indication of tables available for the study of annual trends; other tables have appeared in the Annual Report of the Medical Officer of Health for the City of Birmingham.

Since at the present time the main task is to improve the completeness and accuracy of the returns, no tests of statistical significance have been applied to the results. Detailed breakdowns for a single year, particularly at the present low level of neonatal mortality, very soon result in numbers too small to have any significance. As already stated, the chief value of such a scheme is that it provides for the accumulation of data routinely collected over a period of years.

Discussion of the significance of the results obtained and comparisons with previous published work have been deferred until more detailed and comprehensive studies, based on a further year's material, can be presented. These aspects of neonatal mortality will need further elaboration before practical conclusions can be safely drawn.

Four categories of information about the baby are so far available for study: neonatal mortality, birth weight, congenital malformations, and birth injuries and diseases in the first two weeks of life. Mortality and morbidity after the first month will become available for analysis at a later date. Associated with the foregoing is information which may be classified broadly under four headings:

(1) Demographic, i.e. age of mother, parity, and plurality;

(2) Socio-economic;

(3) Administrative, including information about the work of the department;

(4) Medical. 
All the facts have medical aspects, but the fourth heading covers those tables which are chiefly of interest in providing material for the fatality rates described above. Needless to say, all the data are inter-related and the scheme merely indicates the fields from which the following examples have been selected.

(1) Age of Mother, Parity, and Plurality.-Parity can be treated by recording either the number of previous live births, or the number of previous live and stillbirths. The former is wanted for demographic purposes; the latter is more instructive for analysis of mortality and morbidity. Age of mother and live-birth parity have been tabulated for all resident births and will appear in the City Abstract. Sex by age of mother is also available. 39 per cent. of all resident births were first births. The sex ratio was 106.8 males to 100 females. In the tables of this report the parity referred to is total parity, i.e. the number of previous live and stillbirths.

Before presenting aspects of neonatal mortality, it must be noted that the mortality rates referred to are survivorship rates, i.e. they cover all neonatal deaths occurring among those born in 1949, as distinct from customary rates which relate deaths to births occurring in the same calendar year. Up to 4 weeks, migration is negligible and survivorship rates give a more accurate account in a period of falling birth and death rates, though over a period of years the difference in respect of neonatal mortality can only be small. The neonatal mortality rate for Birmingham births in 1949 obtained in this way was $17 \cdot 3$ per 1,000 live births.

Birmingham births were tabulated simultaneously by age of mother, total parity, and plurality, together with neonatal mortality. Tables by age and parity separately have appeared in the Annual Report. Most subsequent tables were likewise tabulated by age and parity. This enables two things to be done: changes in mortality can be presented in subsequent years with the effect of age and parity removed, and in the current year, mortality rates by socio-economic and other agencies have been standardized for differences in age and parity.

\section{(2) Socio-economic Agencies affecting Neonatal Mortality}

(i) Housing Conditions. - Health visitors were asked to ascertain for every birth the number of persons in the household and the number of rooms occupied by the household. The new baby is included in the household and detailed instructions as to the enumeration of persons and households were issued. Since a trial run disclosed a very high proportion of babies born to households with two or more persons per room, a check survey was carried out in the field on a very small randomly selected sample of such households. The check revealed that difficulties of enumeration were great, since most of the cases were those of two or more households or families sharing a dwelling, rather than one family too big for its house. There was some tendency to describe the living conditions of the baby's immediate family rather than that of the often complex household of which the family might be a part. The effect of so doing was to exaggerate slightly the number of overcrowded cases, but it does not seem likely that errors of this kind greatly distort the picture. The figures are certainly more realistic than those 
commonly presented for overcrowding in which a baby under 1 year is customarily omitted, a grotesque procedure considering the numerous situations which arise when a baby is born to a married couple living in one room. In so far as a comparison with overcrowding statistics for the city compiled on the customary basis at an earlier date is possible, there is a suggestion that those families with new babies are especially hard hit by the current housing shortage. This is what one would expect, since they are on the whole younger and less likely to be established in a prewar house. Babies that died in hospital gave rise to some ambiguity. Usually such babies were omitted from the count of persons and reasonably so, but this led to somewhat fallacious mortality rates. In the table which follows, the dead baby is included in the household. The effect on total proportions in the various housing groups shown in Table II is negligible. Mortality rates standardized for age and parity and adjusted for deaths where housing conditions are unknown are also shown.

TABLE II

Standardized Neonatal Mortality Rates, by Number of Persons Per Room

\begin{tabular}{|c|c|c|c|c|}
\hline Housing Group & & & $\begin{array}{l}\text { Percentage of } \\
\text { all Live Births }\end{array}$ & $\begin{array}{c}\text { Standardized } \\
\text { Neonatal } \\
\text { Mortality Rate }\end{array}$ \\
\hline $\begin{array}{l}\text { Less than one person per room } \\
\text { One and less than } 1.5 \text { persons per room } \\
1.5 \text { and less than two persons per room } \\
\text { Two or more persons per room }\end{array}$ & $\begin{array}{ll}\ldots & \cdots \\
\cdots & \cdots \\
\cdots & \cdots \\
\ldots & \cdots\end{array}$ & $\begin{array}{l}\cdots \\
\cdots \\
\cdots \\
\cdots\end{array}$ & $\begin{array}{l}23 \cdot 5 \\
33 \cdot 0 \\
20 \cdot 1 \\
23 \cdot 4\end{array}$ & $\begin{array}{l}15 \cdot 8 \\
16 \cdot 0 \\
17 \cdot 2 \\
18 \cdot 3\end{array}$ \\
\hline
\end{tabular}

The mothers living in the most overcrowded conditions fall into two groups: young mothers with a first or second child, and mothers of 35 or over with three or more previous children. The first group represents the young families unable to get a house and obliged to share or live in rooms, and the second group includes families too large for their accommodation. The number of deaths in a single year is too small to permit of any significant breakdowns by cause of death, but birthinjuries are more frequent in the most overcrowded group, and congenital malformations in the best-housed group.

Seeing that most neonatal deaths occur within a few days after birth and are largely determined pre-natally or at the time of birth, one would not expect the connexion with housing conditions to show more than the relatively slight gradient of Table II. The effect on deaths after 4 weeks is likely to be more direct. Analysis of later deaths on the lines of Table II is not yet available, but a comparison can be made between the proportion of births in the most overcrowded group by Welfare Centre areas, and the average infant mortality for the past three years in the same area (Figs 1 and 2). The product-moment correlation co-efficient $(0 \cdot 79)$ is almost certainly significant in spite of the small number of areas (31). The most overcrowded areas form a solid block in and around the "Central Areas" (scheduled for re-development). Comparison of the three worst areas (i.e. those with the highest proportions of households with two or more persons per room) 


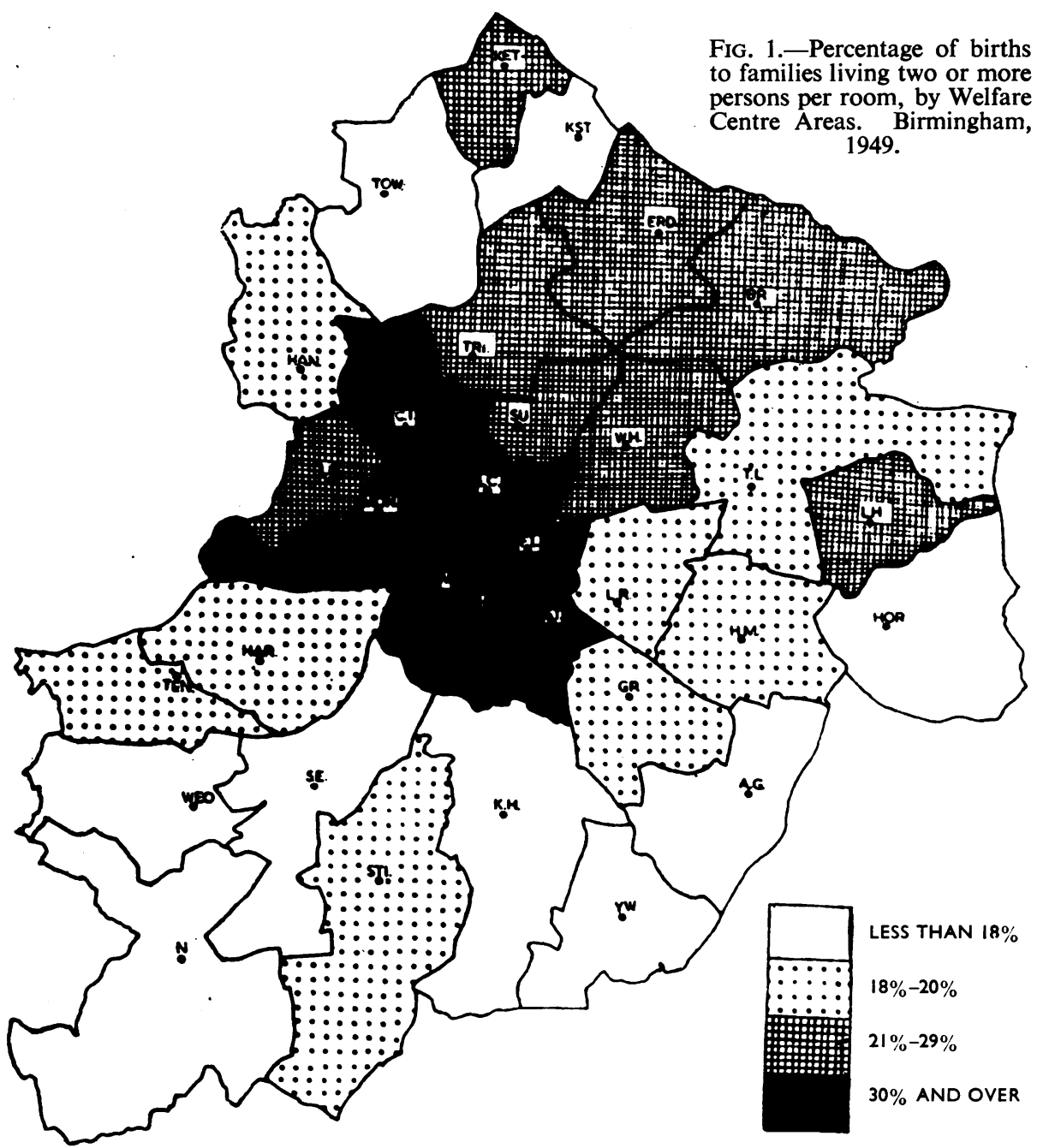

with the three best areas, shows that average infant mortality has been 67 per cent. higher in the former than in the latter. As many previous studies have shown, the relation of overcrowding to infant mortality is not a simple one. The overcrowding shown in the centre of the city is linked with comparatively low wages, derelict buildings, and a whole interlinked complex of social conditions. All that the foregoing maps and figures can do is to pin-point an area of the city where, in spite of all recent social advances, there is a section of the population living under conditions detrimental to the welfare of their children. As both maps are descriptions of social areas, it seems legitimate to compare the deaths for the past three years with the housing conditions of new babies.

(ii) Employment of Women during Pregnancy.-Analysis by age and parity is 


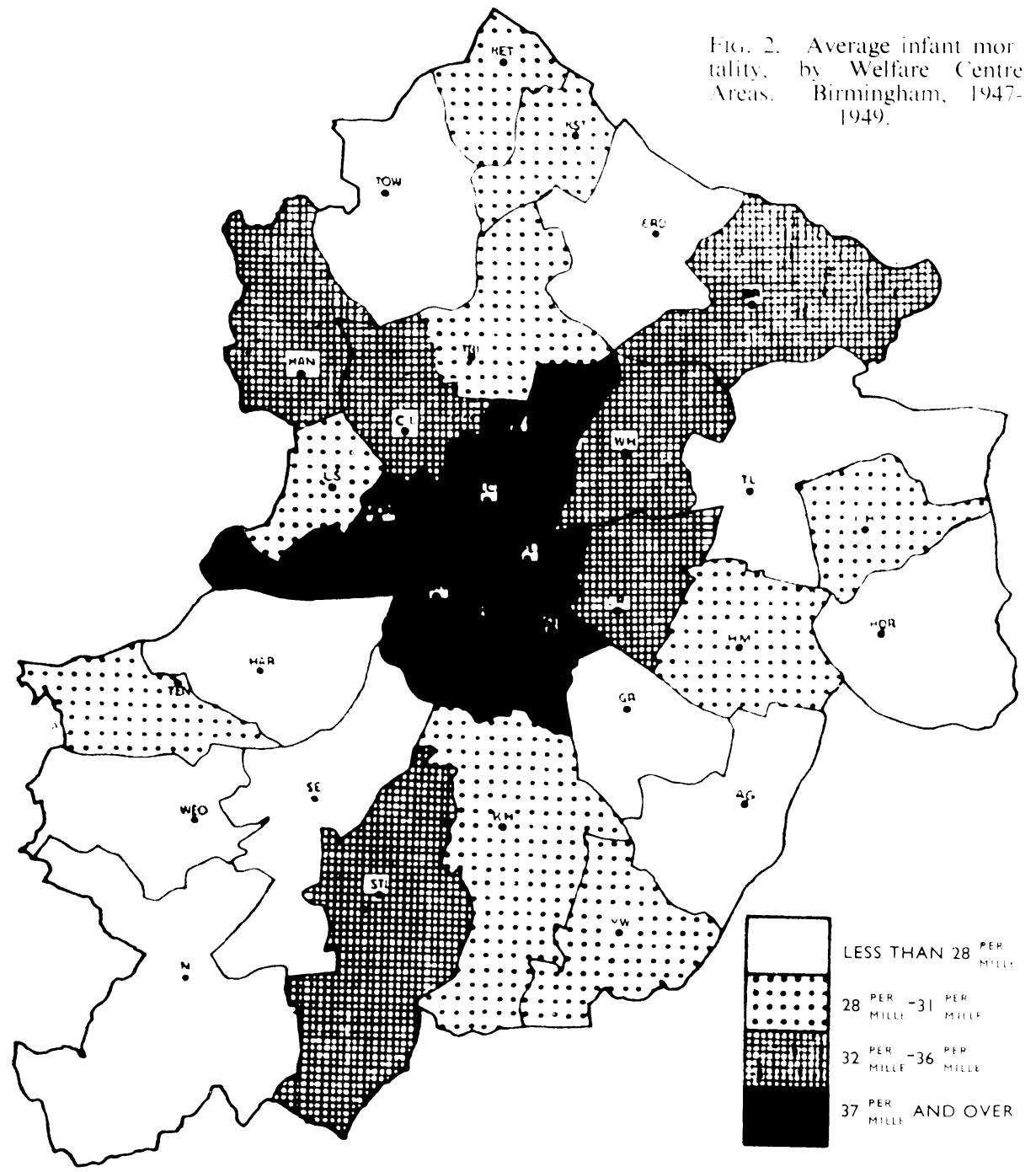

particularly necessary in studying the effect of employment of women in pregnancy, since, as Table III shows, the proportion working is very much greater for primiparae. In what follows, the unknown cases have been included with working mothers in order to obtain a clear-cut group of mothers known not to have been gainfully employed. The standardized neonatal mortality rate for working mothers is 21.8 as compared with 16.9 for non-working mothers. On further analysis, however, it appears that mothers gainfully employed in their first pregnancy experience lower neonatal mortality rates than those not so employed, suggesting that the adverse effects of gainful employment are associated with poverty inducing mothers of several children to go out to work. 
That the number of unknown deaths does not seriously distort the picture is indicated by the fact that the proportion of immature babies $\left(5 \frac{1}{2} \mathrm{lb}\right.$. or less) follows the same pattern as that of neonatal deaths. So also do the proportions of mothers with some antenatal disease or complication. Neonatal mortality rates among mothers with no antenatal disease or complication are again lower for working mothers in first pregnancies and higher for other parities, but the difference is much less than when all mothers are taken together.

TABLE III

Gainful Employment of Mothers by Parity*

\begin{tabular}{|c|c|c|}
\hline Parity & & $\begin{array}{l}\text { Percentage of Mothers } \\
\text { in Gainful Employment }\end{array}$ \\
\hline $\begin{array}{lc}\text { Primiparae } & . . \\
\text { 2nd parae } & . . \\
\text { 3rd parae } & . \\
\text { 4th parae } & . \\
\text { 5th parae } & . \\
\text { 6th parae and over }\end{array}$ & $\begin{array}{ll}\ldots & \ldots \\
. & \ldots \\
. & \cdots \\
. & \ldots \\
\cdots & \cdots \\
\ldots & \cdots\end{array}$ & $\begin{array}{l}61 \cdot 3 \\
19 \cdot 9 \\
15 \cdot 3 \\
13 \cdot 7 \\
16 \cdot 1 \\
12 \cdot 3\end{array}$ \\
\hline All parities & $\begin{array}{ll}\ldots & \ldots\end{array}$ & $33 \cdot 6$ \\
\hline
\end{tabular}

(3) Administrative Aspects of Neonatal Mortality.-The main features of public health work which the present material can illuminate are antenatal care, hospitalization, and the care of immature infants. The last has already been the subject of intensive study and a detailed study of the first two would be mainly of local interest. For illustrative purposes a brief account of neonatal mortality in relation to the amount of antenatal care is presented.

Neonatal mortality rates standardized by age and parity and adjusted for unknown deaths are shown in Table IV. It has to be remembered that the amount of antenatal care was unknown for 11 per cent. of all births. Clinic patients comprise those who have attended a Corporation Clinic at least once, but the number of attendances recorded may include visits to hospitals, doctors, etc.

TABLE IV

Standardized Neonatal Mortality Rates by Amount of Antenatal Care

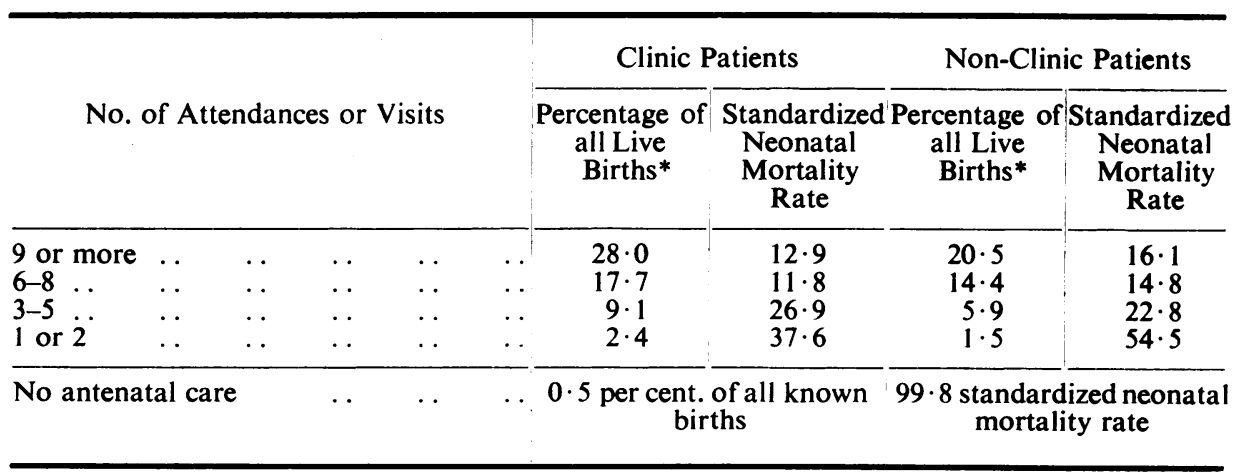

* Excluding cases with amount of antenatal care unknown. 
Deaths in the group with no antenatal care are almost entirely illegitimate births (6 out of 8 ) and are thus self-explanatory. The exclusion of illegitimate births and deaths would not affect the differences between the four groups above with the larger number of attendances on the one hand and the four with the lowest on the other. Again, one might surmise that deaths associated with a short period of gestation might swell the numbers in the groups with fewer attendances. This circumstance, however, could hardly affect the very high mortality rates for mothers with only one or two attendances. The amount of antenatal care is greatest for first and second children of mothers between 30 and 40 . The slightly lower percentages for younger mothers may reflect higher proportions illegitimate. There is a definite falling off after the second child.

It is not suggested that there is a simple causal relationship between the number of antenatal attendances and neonatal mortality. The display of necessary initiative on the part of the mother is undoubtedly associated with social and personal characteristics contributing to the welfare of the family. In future years the accumulation of larger numbers will permit more detailed breakdowns and may throw light on the circumstances in which pregnant women fail to make use of the services available to them.

\section{(4) Medical Aspects of Neonatal Mortality}

(i) Antenatal Record.-When records cover a greater part of the population, the relation between the mother's health in pregnancy and the condition of the baby may prove a fruitful field of research. The antenatal record was available for 83 per cent. of all live births. A table has already appeared showing the incidence of certain diseases and complications of pregnancy and the corresponding neonatal mortality rates. In 19.9 per cent. of births with known records, some disease or complication occurred, and the neonatal mortality rate was more than four times as high for these births as for those preceded by an uncomplicated pregnancy. Of separately coded complications, the most frequent was toxaemia $(1,085)$.

The medical record can now be related to the amount of antenatal care. Cases of Rhesus-negative mothers with agglutinins present are not included in this section.

There is a small but significantly higher proportion of women with antenatal disease or complication among those with nine or more attendances compared with the rest with known antenatal care. This is true of each complication severally and for both primiparae and multiparae, and the difference probably accounts for the slightly higher mortality rates for mothers with nine or more antenatal attendances or visits compared with those having six to eight attendances.

However, as Table V (opposite) shows, the neonatal mortality rates are higher among those with fewer attendances for women with some complication and for those with none. The excess deaths are especially frequent among multiparae with some complication and inadequate antenatal care. Table $\mathrm{V}$ is based on 14,922 live births. The excess mortality associated with inadequate antenatal care is chiefly seen among multiparae with toxaemia. 
(ii) Duration of Labour.-No exact determination of the moment at which labour begins is possible, yet it seems that with sufficient numbers of observations, the data grouped within quite wide time limits might be of significance. In particular, prolonged labour is frequently regarded as a contributory cause of neonatal mortality. To avoid the fallacy of arguing backwards from a death to an arbitrarily selected previous event, it seemed worth while examining the mortality associated with different durations of labour.

Hours of labour are recorded for 77 per cent. of all first births, and for 81 per cent. of all later births. They were also known for 81 per cent. of the deaths. Values recorded oscillate in a somewhat incomprehensible manner between odd and even numbers. So the data were tabulated by 2 -hour duration groups up to 29 hours, and by 10-hour duration groups thereafter. The first group, which includes cases with 0 hours labour, is of somewhat dubious validity and so is the last group of $\mathbf{9 0}$ hours or more. The former contains at least one Caesarean, and the latter one case said to have been of 129 hours. The very short durations include deliveries which occurred before the arrival of doctor or midwife, where the duration is largely guesswork.

TABLE $V$

Antenatal Diseases and Complications

NEONATAL MORTALITY RATES, BY AMOUNT OF ANTENATAL CARE

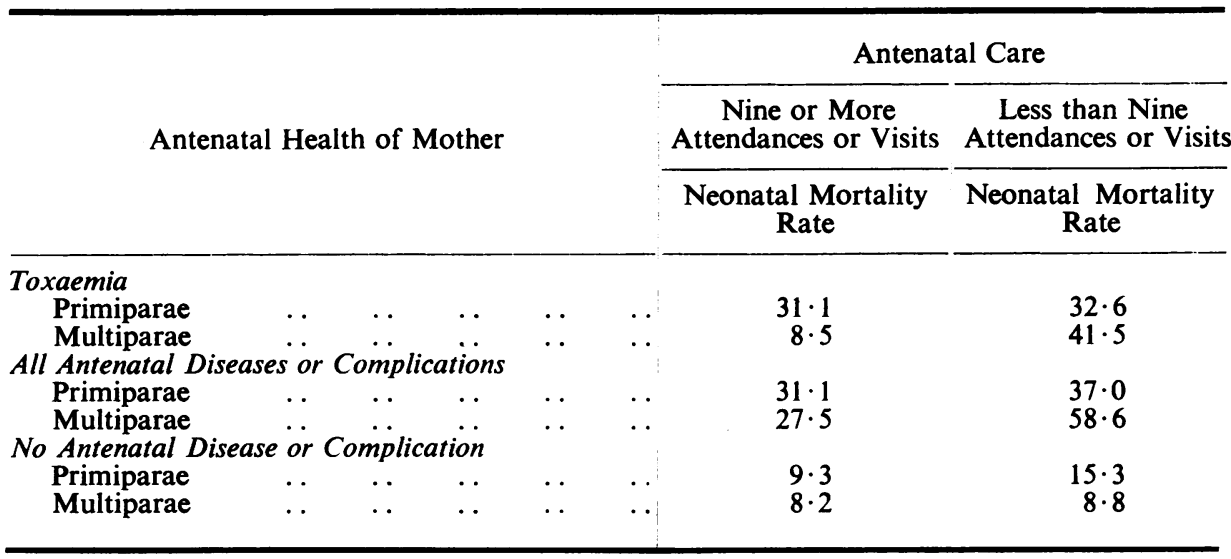

The distributions for primiparae and multiparae are naturally very different. The former have a peak at 10 hours, the latter at 5 hours. Further, a much larger proportion of multiparae have durations at or near the peak value, so that the proportion with very long durations is considerably less. Both distributions are markedly skew. Table VI (overleaf) shows the percentage incidence.

Owing to the small numbers, death-rates by duration are very unstable, but when the 2-hour duration groups are smoothed by 3-period moving averages, a fairly steady trend is apparent which is very similar for both primiparae and multiparae.

Both agree in showing high death rates for very short durations falling to a 
TABLE VI

Duration of Labour, Percentage Incidence

\begin{tabular}{|c|c|c|}
\hline \multirow{2}{*}{$\begin{array}{c}\text { Duration of } \\
\text { Labour } \\
\text { (hours) }\end{array}$} & \multicolumn{2}{|c|}{ Percentage of all Live Births* } \\
\hline & Primiparae & Multiparae \\
\hline Less than 2 & .5 & 3.2 \\
\hline $2-3$ & $3 \cdot 3$ & 13.0 \\
\hline $4-5$ & $6 \cdot 8$ & $17 \cdot 2$ \\
\hline $6-7$ & $9 \cdot 6$ & $15 \cdot 2$ \\
\hline $8-9$ & 10.1 & 12.6 \\
\hline $10-11$ & $10 \cdot 7$ & $9 \cdot 4$ \\
\hline $12-13$ & $9 \cdot 4$ & $7 \cdot 3$ \\
\hline $14-15$ & $8 \cdot 1$ & $5 \cdot 5$ \\
\hline $16-17$ & $6 \cdot 1$ & 3.9 \\
\hline $18-19$ & $6 \cdot 2$ & $3 \cdot 1$ \\
\hline $20-21$ & $4 \cdot 7$ & $2 \cdot 3$ \\
\hline $22-23$ & $4 \cdot 3$ & $1 \cdot 5$ \\
\hline $24-25$ & $3 \cdot 4$ & $1 \cdot 3$ \\
\hline 26-27 & $2 \cdot 5$ & 0.9 \\
\hline $28-29$ & $2 \cdot 3$ & 0.6 \\
\hline $30-39$ & 1.0 & 0.3 \\
\hline $40-49$ & 0.6 & 0.1 \\
\hline $50-59$ & $0 \cdot 3$ & 0.1 \\
\hline $60-69$ & $+\{0.2$ & 0.1 \\
\hline $70-79$ & 0.1 & - \\
\hline $80-89 \quad \ldots$ & $0 \cdot i$ & - \\
\hline 90 and over & $0 \cdot 1$ & - \\
\hline
\end{tabular}

* Omitting births with duration of labour unknown.

$\dagger$ One-fifth of incidence for all durations over 10-hour period.

minimum at about 8 hours. Thereafter, the death rates of primiparae rise gradually while those of multiparae remain about the same. Both again agree in showing a further conspicuous rise for durations of 23 hours and over. As the trends are so similar, all parities are combined in Table VII.

A breakdown by cause of death involves still smaller numbers and can only be suggestive. The death rate from prematurity alone is high for very short durations. Deaths attributed to injury at birth increase rapidly from 23 hours onwards. So also do deaths attributed to congenital malformations.

It is evident that when the optimum period is passed the longer the labour the greater the risk to the baby. The results obtained suggest that durations of more than 22 hours or less than 3 hours might be regarded as contributing significantly to a greater risk of neonatal death.

TABLE VII

Neonatal Mortality Rates by Duration of labour (SIX-HOURLY MOVING AVERAGES, ALL PARITIES)

\section{CONDItION OF BABY}

(i) Congenital Malformations.-As stated above, the number of incomplete returns in respect of the baby's condition at birth and during the first two weeks detracts considerably from the value of the results obtained. The 1949 figures in this section are therefore presented without analysis as an indication of the kind of material which

\begin{tabular}{cc}
\hline Central Duration (hours) & Neonatal Mortality Rate \\
\cline { 2 - 2 } $2 \frac{1}{2}$ & $21 \cdot 5$ \\
$4 \frac{1}{2}$ & $16 \cdot 3$ \\
$6 \frac{1}{2}$ & $14 \cdot 4$ \\
$8 \frac{1}{2}$ & $12 \cdot 5$ \\
$10 \frac{1}{2}$ & $13 \cdot 8$ \\
$12 \frac{1}{2}$ & $15 \cdot 7$ \\
$14 \frac{1}{2}$ & $16 \cdot 6$ \\
$16 \frac{1}{2}$ & $18 \cdot 2$ \\
$18 \frac{1}{2}$ & $16 \cdot 8$ \\
$20 \frac{1}{2}$ & $18 \cdot 5$ \\
$22 \frac{1}{2}$ & $20 \cdot 5$ \\
$24 \frac{1}{2}$ & $24 \cdot 0$ \\
$26 \frac{1}{2}$ & $29 \cdot 3$ \\
30 and over & $25 \cdot 0$ \\
\hline
\end{tabular}


will become available for study as greater completeness and accuracy are achieved. Table VIII shows the incidence of congenital malformations as far as they are known.

The data of Table VIII should prove valuable in future years since they show the incidence of minor malformations and defects over a large population of births. For example, eleven cases of polydactyly were recorded, most of which would probably escape notice for statistical purposes at a later age. Some of the cases of spina bifida and mongolism may not have been confirmed by a specialist diagnosis, but the follow-up at 12 months will enable such records to be confirmed or otherwise. In accord with previous work, more than half the cases of mongolism were born to mothers aged 40 years and over.

(ii) Birth Weight.-In contradistinction to the material of the previous section, birth weight is known for virtually the entire population of births. The conditions under which weighing is done, particularly when midwives are single-handed, are not such as to permit of great accuracy. On account of the lumping together at quarter and half pounds, weights were grouped in half-pound intervals and the numbers in each class tabulated by age of mother and parity. In addition to the special problems of the infant weighing $5 \frac{1}{2} \mathrm{lb}$. or less, it seemed that a comparison of babies' weights from year to year might be of interest. To this end,

TABLE VIII

INCIDENCE of CONGENital Malformations

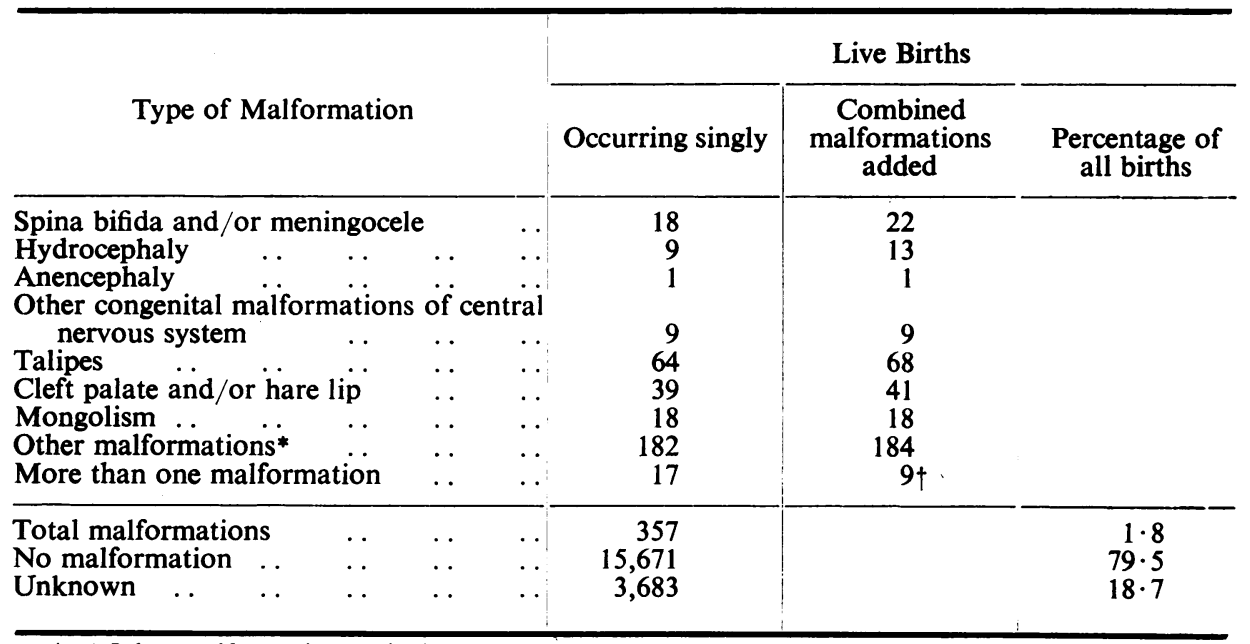

* "Other malformations " include:

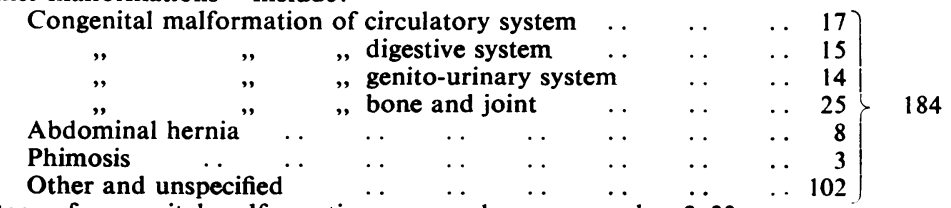

Percentage of congenital malformations among known records $=2 \cdot 22$

$\dagger$ Defects unspecified. 
average birth weights for condensed age and parity classes, distinguishing between single and multiple births, have been computed. Similar tables in subsequent years will enable any changes in average birth weight to be noted, free from the effect of changing age, parity, or proportion of multiple births. Average weights were obtained by regarding all weights in a half-pound class as concentrated at the mid-point of the class. Average weights obtained by fitting a curve to the crude data differed only negligibly from those obtained in this way, so that the considerable extra arithmetic involved in the more refined process did not seem to be necessary.

\section{Summary}

(1) A description is given of the record system introduced into the Maternity and Child Welfare Department of the City of Birmingham. The health visitors' Record Card for all live births, with parallel cards for neonatal deaths and eventually for stillbirths, has been re-designed so as to provide a continuous history from the antenatal period up to the age of 5 years. The principal occurrences are recorded in such a way that the information can be readily transferred to punch cards.

(2) While the first complete year must be regarded as still partly experimental, sufficient has been accomplished to indicate the value of the material in subsequent years, and some of the results are presented as an indication of the scope of the material.

(3) Neonatal mortality rates by age of mother, parity, and plurality have been computed. It will thus be possible to examine trends in mortality free from the influence of extraneous factors.

(4) Standardized neonatal mortality rates are presented for certain socioeconomic agencies. Higher mortality was found to be associated with overcrowding and with the gainful employment of mothers who already have at least one child.

(5) Inadequate antenatal care was found to be associated with considerably higher standardized neonatal mortality rates. This was true both for primiparae and multiparae and for mothers with and without an uncomplicated pregnancy.

(6) Fatality rates associated with antenatal complications and with varying durations of labour are presented.

(7) Table VIII shows, as far as was ascertainable, the incidence of congenital malformations of all types recognizable at birth. Average birth weights have been computed for age and parity classes, distinguishing single and multiple births, with a view to annual comparisons.

\section{APPENDIX}

\section{SUMMARY OF FIELDS OF INFORMATION}

The following items are transferred to punch cards from the Health Visitor's Record reproduced in Figs 4-6 (overleaf). The card formerly in use is shown in Fig. 3 (opposite) for comparison. 

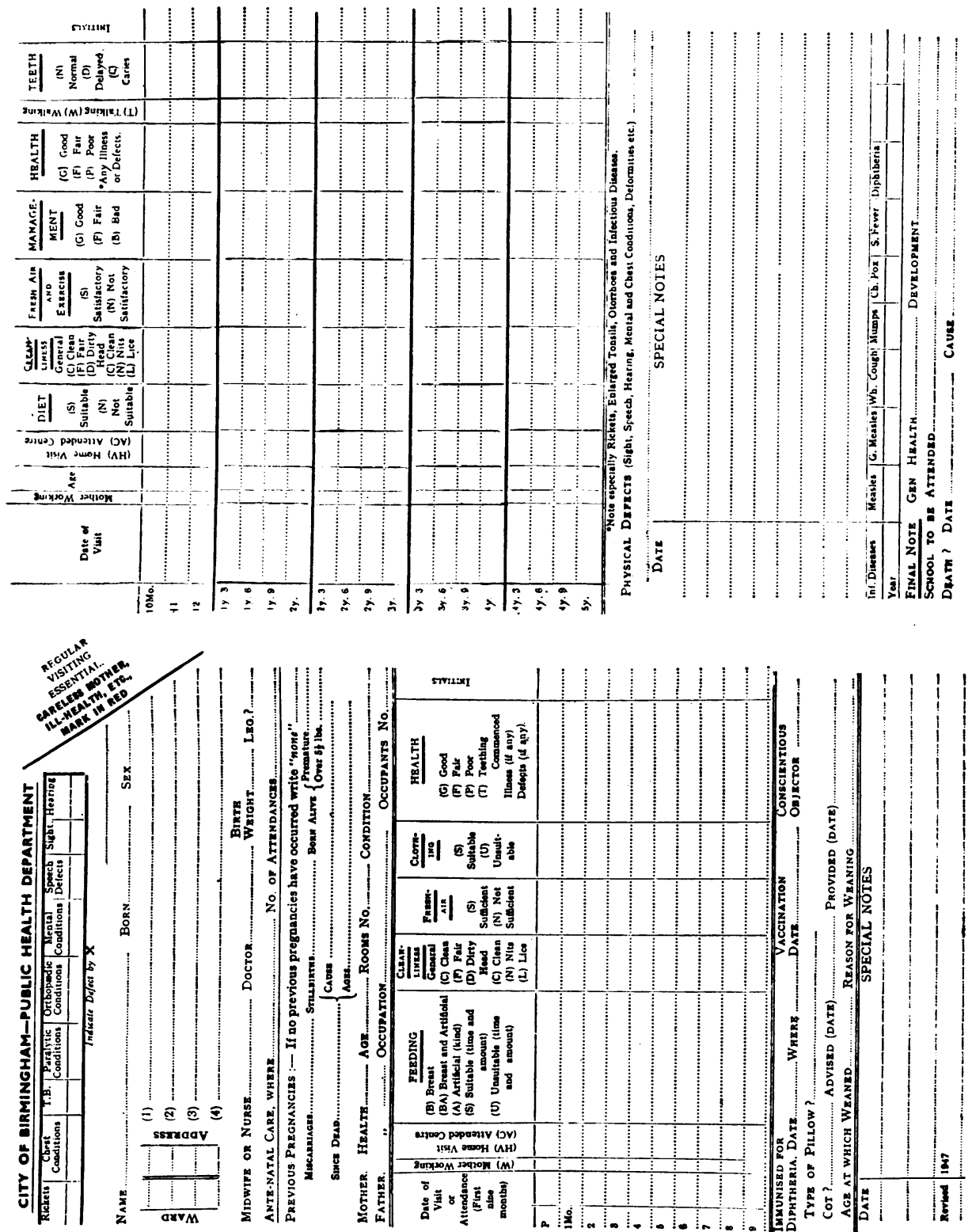

FIG. 3.-Health Visitor's Record, 1948. Maternity and Child Welfare Department, City of Birmingham. 


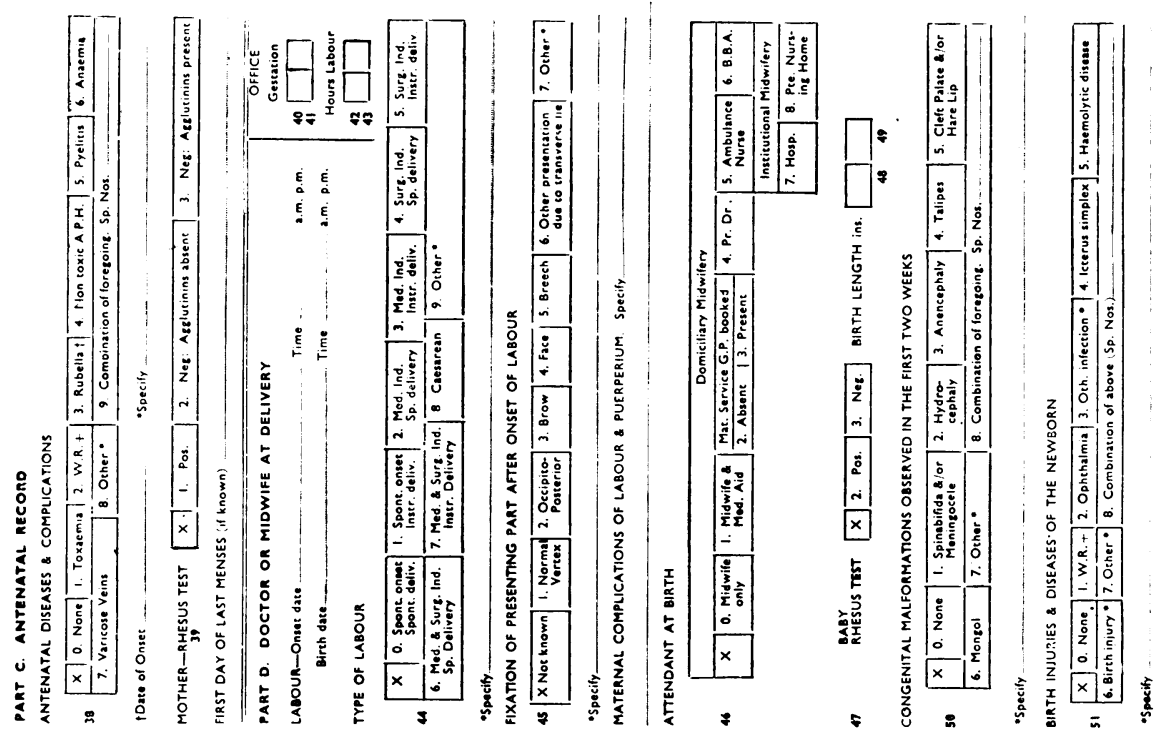

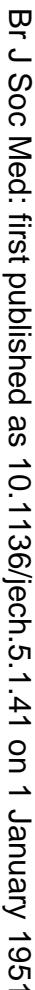

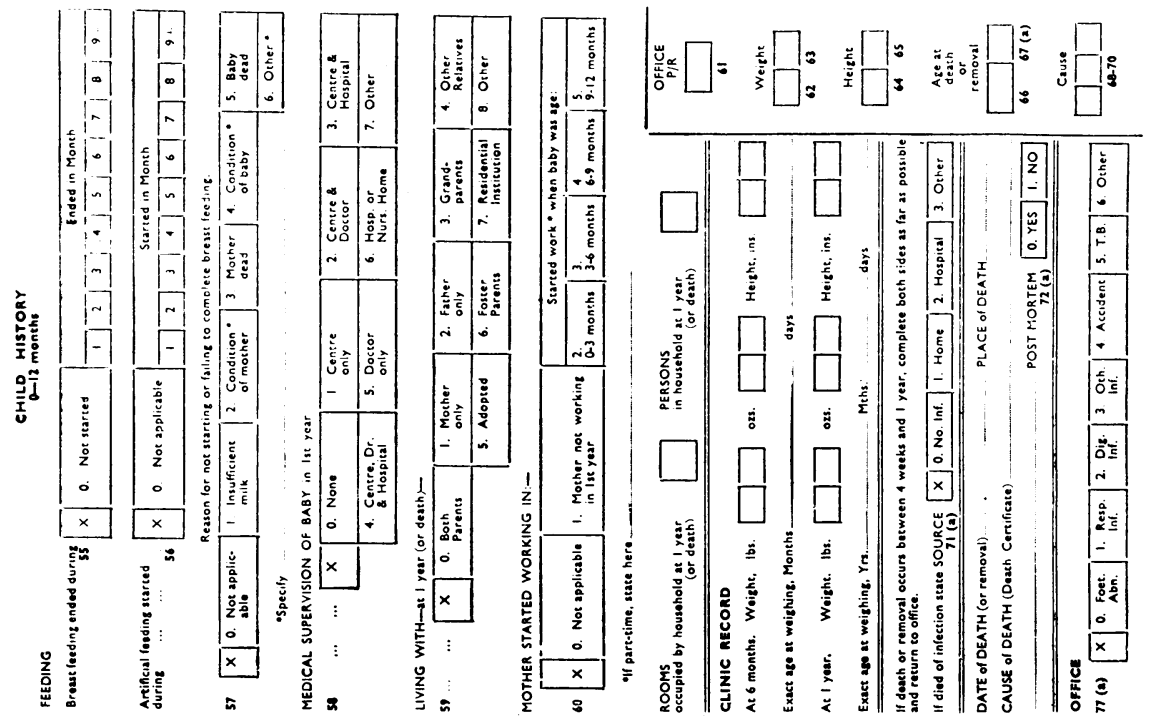

FIG. 4.- Health Visitor's Record, 1950 (page 1, front and back). Maternity and Child Welfare Department, City of Birmingham. 

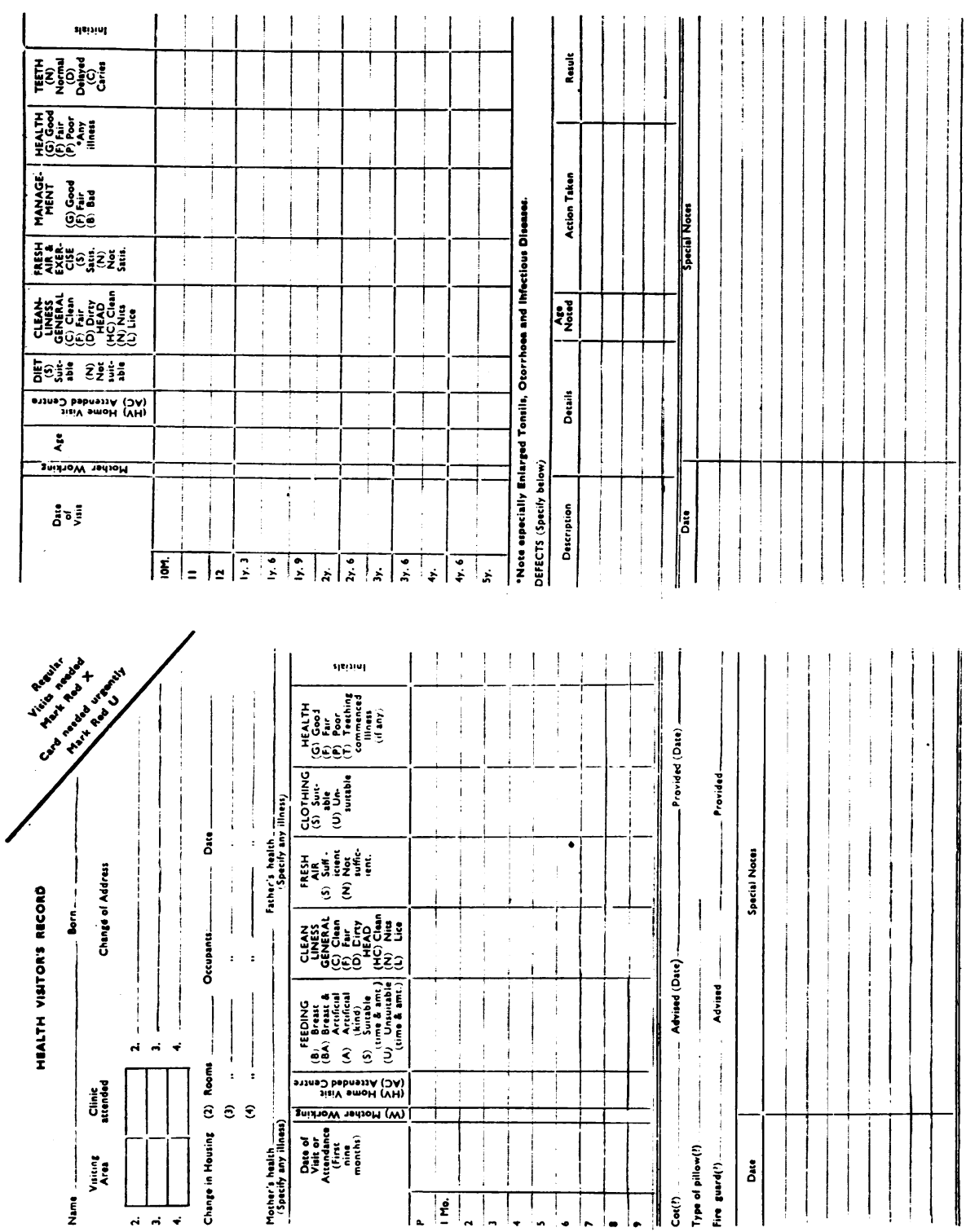

Fig. 5.-Health Visitor's Record, 1950 (page 2, front and back). 

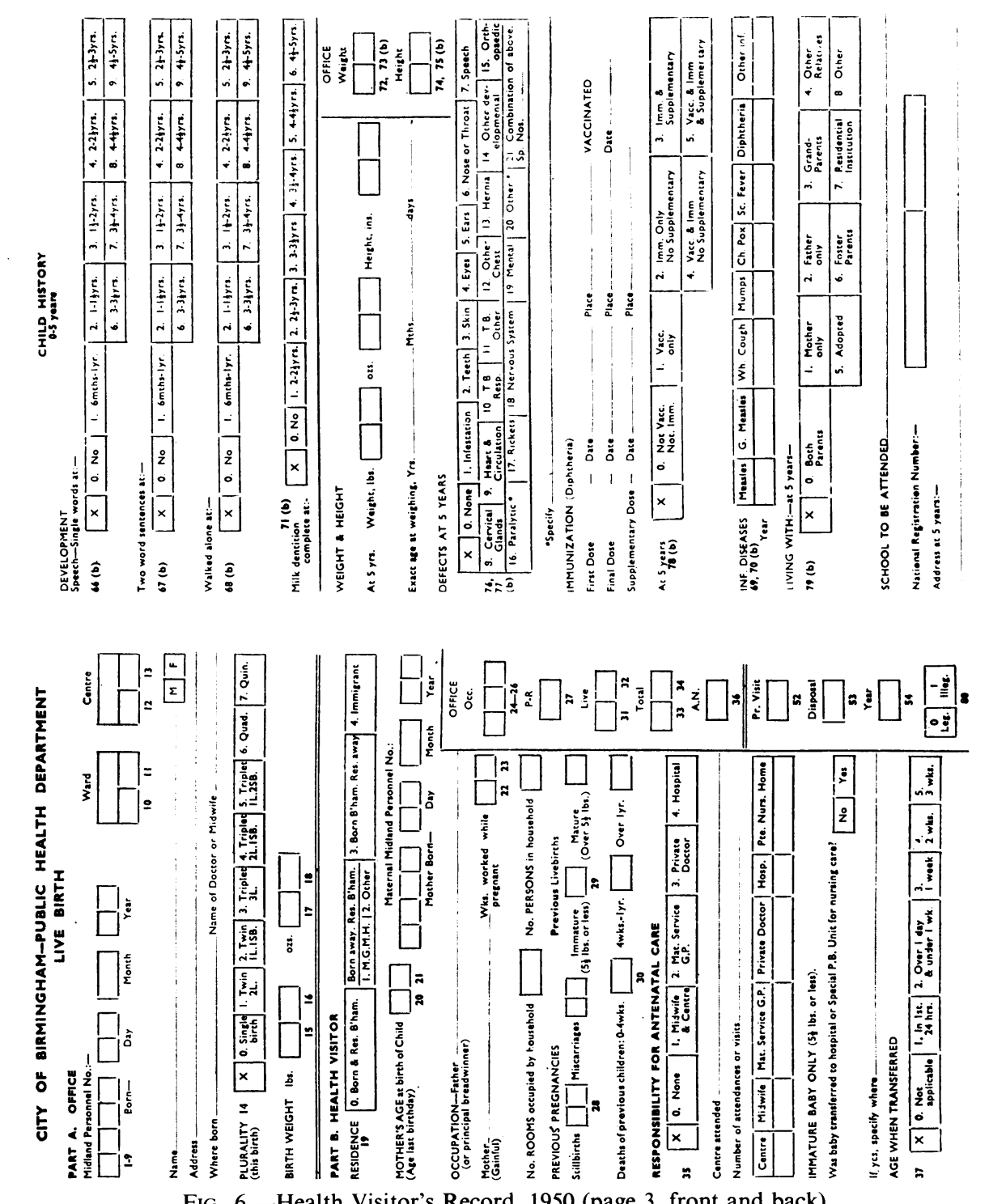

FIG. 6.-Health Visitor's Record, 1950 (page 3, front and back). 
(i) Midland Personnel Number. This is a 9-column personal code number originated by the Department of Medical Statistics in the University of Birmingham. It embodies date of birth and sex for which 6 columns would be required in any case. It also permits of the cards being sorted back into the filing order required by the office after a series of Hollerith tabulations. In the future it is expected to facilitate collation with hospital documents and the work of other departments.

(ii) Residence (Birmingham or out-of-city, ward, centre).

(iii) Maternal age, previous live and stillbirths, premature births, neonatal deaths, plurality.

(iv) Occupation of father and principal breadwinner, persons per room.

(v) Weeks of employment during pregnancy, antenatal care, antenatal diseases and complications. Maternal Rhesus test.

(vi) Period of gestation, attendant at birth, duration and type of labour, presentation.

(vii) Birth weight, length, congenital malformations, neonatal diseases and birth injuries, baby's Rhesus test.

(viii) Premature care.

(ix) At one year: family status (living with both parents, adopted, etc.), employment of mother, persons per room, weight, height.

(x) Developmental history: breast feeding, speech, walking, dentition.

(xi) At 5 years: family status, persons per room, weight, height, defects, infectious diseases, vaccination and immunization.

(xii) Deaths. Relevant portions as above, and age at death, cause of death, revised cause of death, post-mortem findings, breast feeding, source of infection, medical supervision. 